\title{
Role of Retrograde His Purkinje Block in the Initiation of Supraventricular Tachycardia by Ventricular Premature Stimulation in the Wolff-Parkinson-White Syndrome
}

\author{
Masood Akhtar, Mohammad Shenasa, and Donald H. Schmidt, \\ Natalie and Norman Soref and Family Electrophysiology Laboratory, \\ University of Wisconsin Mount Sinai Medical Center, \\ Milwaukee, Wisconsin 53201
}

A B S TRACT The precise mechanisms for paroxysmal reentrant supraventricular tachycardia (PSVT) initiation during right ventricular premature stimulation $\left(\mathrm{V}_{2}\right.$ method) were analyzed in 14 consecutive patients with Wolff-Parkinson-White Syndrome in whom the PSVT was inducible during retrograde refractory period studies. 9 patients had left-sided and the remaining 5 of 14 had right-sided ventriculo-atrial (VA) accessory pathway (AP). At the basic cycle lengths $\left(\mathrm{V}_{1} \mathrm{~V}_{1}\right)$ ranging from 550 to $900 \mathrm{~ms}$ (mean, 657.1 \pm 139.5 ), closely coupled $\mathrm{V}_{2}$ (mean $\mathrm{V}_{1} \mathrm{~V}_{2}, 357.3 \pm 59.2 \mathrm{~ms}$, range $320-500$ ) produced retrograde $\mathrm{His}$ bundle $\left(\mathrm{H}_{2}\right)$ activation via the bundle branches and retrograde atrial $\left(\mathrm{A}_{2}\right)$ activation via the AP. As the $V_{1} V_{2}$ were further shortened, the $V_{2}$ showed a retrograde block in the His Purkinje system (HPS) and conducted to the atria via AP in 9 of 14 cases. Subsequently, the $\mathrm{A}_{2}$ impulse conducted anterograde over the atrioventricular node-HPS to initiate a PSVT or an atrial echo response in all nine cases. In none of the patients was a PSVT induced by $\mathrm{V}_{2}$ when the latter produced retrograde $\mathrm{H}_{2}$ activation via the bundle branches. In 10 of 14 cases, however, the retrograde $\mathrm{H}_{2}$ was followed by a $V_{3}$, due to macroreentry in the HPS. The $\mathrm{V}_{3}$ in turn blocked retrogradely in the HPS while producing $\mathrm{A}_{3}$ via the AP to initiate a PSVT or an atrial echo response in 9 of 10 cases. Retrograde block of $V_{2}$ and/or $\mathrm{V}_{3}$ in the HPS resulted in PSVT initiation in 13 of 14 cases, whereas in the remaining 1 case the exact mechanism was not clear. In none of the patients in this series was the PSVT initiated with a retrograde block of

Address reprint requests to Dr. Masood Akhtar, Cardiovascular Disease Section, Mount Sinai Medical Center, PO Box 342, Milwaukee, Wisc. 53201.

Received for publication 22 August 1980 and in revised form 7 November 1980 .
$\mathrm{V}_{2}$ in the atrioventricular node with or without concomitant retrograde $\mathrm{A}_{2}$ activation via the AP. We conclude that within the ranges of cycle lengths tested, a retrograde block of $\mathrm{V}_{2}$ and/or $\mathrm{V}_{3}$ in the HPS is the most common mechanism for initiation of PSVT during ventricular premature stimulation in patients with the Wolff-Parkinson-White Syndrome.

\section{INTRODUCTION}

Paroxysmal reentrant supraventricular tachycardia (PSVT) ${ }^{1}$ utilizing atrioventricular (AV) node and His Purkinje system (HPS) anterogradely, and an accessory pathway (AP) in the retrograde direction, is relatively common in patients with the Wolff-Parkinson-White (WPW) Syndrome (1-4). Several studies have demonstrated that PSVT in these patients can be initiated with atrial as well as ventricular premature beats. It has also been previously demonstrated that during ventricular premature stimulation, the ability or inability to induce PSVT depends upon the various patterns of ventriculo-atrial (VA) conduction along the normal and the AP (5-8). Although addressing themselves to the VA conduction along the normal pathway in patients with the WPW Syndrome, studies to date have not made a clear distinction between conduction along the HPS vs. the AV node, the two components of normal pathway. Since the HPS and AV node have different electrophysiological properties and therapeutic responses, a clear understanding of the role of retrograde conduction in

${ }^{1}$ Abbreviations used in this paper: Ae, atrial echo; AP, accessory pathway; AV, atrioventricular; ERP, effective refractory period; HPS, His Purkinje system; PSVT, paroxysmal rentrant supraventricular tachycardia; VA, ventriculo-atrial; WPW, Wolff-Parkinson-White. 
the HPS and the AV node in the initiation of PSVT is desirable. This report demonstrates the role of retrograde block within the HPS in the initiation of PSVT during right ventricular premature stimulation in 14 patients with the WPW Syndrome.

\section{METHODS}

Right heart catheterization was performed in a post absorptive, nonsedated state, using local anesthesia. The nature of the procedure was explained to all patients and signed consents were obtained. Quadripolar electrode catheters were percutaneously inserted into peripheral veins and positioned in the region of the high right atrium, $\mathrm{AV}$ junction, coronary sinus, and right ventricle. The catheters were used for local bipolar electrogram recordings and/or electrical stimulation using techniques previously described (9). Surface electrocardiogram Leads I, II, and VI, intracardiac electrograms, and time lines were simultaneously displayed on a multichannel oscilloscope and recorded on a frequency-modulated tape. The records were subsequently retrieved for analysis at a paper speed of $100-150 \mathrm{~mm} / \mathrm{s}$. Intracardiac stimulation was performed using digital stimulator capable of delivering rectangular impulses with adjustable voltage and duration. During the conduct of these studies, patient isolation existed and all equipment was carefully grounded.

Retrograde refractory period studies were performed using ventricular extrastimulus method. Premature stimuli were introduced after eight ventricular paced beats of predetermined constant cycle length. The coupling intervals were gradually reduced by $10-20 \mathrm{~ms}$ until ventricular muscle refractoriness was encountered.

Definition of terms. A complete set of definitions for anterograde and retrograde conduction and refractory period studies has been previously published (9). Pertinent definitions repeatedly used in this text will be outlined here.

The A-H interval was measured from the onset of low atrial electrogram to the beginning of His bundle potential. The $\mathrm{H}-\mathrm{V}$ and RB-V intervals were measured from the onset of His and right bundle branch deflections to the earliest detectable ventricular activity, either on the surface electrocardiogram or the local intracardiac tracings.

Atrial echo (Ae) response represents spontaneous retrograde excitation of the atrium exclusively via the accessory pathway occurring during nonpaced beats.

For an easier comprehension some of the terms used and electrophysiologic parameters measured during retrograde refractory period studies are graphically depicted in Fig. 1. The $S_{1}, V_{1}$, and $A_{1}$ represent stimulus artifact, ventricular, and retrograde atrial deflections, respectively, during the basic drive, whereas $S_{2}, V_{2}, H_{2}$, and $A_{2}$ represent stimulus artifact, ventricular, His bundle, and atrial deflections following the premature beat.

The retragrade activation in response to $V_{2}$ of the $\mathrm{H}_{2}$ via the bundle branches and the $A_{2}$ via AP is shown in panel A. Exclusive VA conduction via the AP during both $V_{1}$ and $V_{2}$ is suggested by the sequence of atrial activation and its lack of change following $\mathrm{V}_{2}$ despite significant delay along the normal pathway $\left(\mathrm{V}_{2} \mathrm{H}_{2}\right.$ interval).

The effective refractory period (ERP) of AP (panel B) is the longest $V_{1} V_{2}$ where $V_{2}$ blocks in the AP.

The ERP-AV node (panel C) is the longest $\mathrm{V}_{1} \mathrm{H}_{2}$ where $\mathrm{H}_{2}$ is not followed by $A_{2} . V_{1} H_{2}$ is taken in lieu of retrograde $H_{1} H_{2}$ since $H_{1}$ is generally not visible during the basic drive, and it is assumed that $\mathrm{V}_{1} \mathrm{H}_{1}$ interval is constant (9). It should be noted that ERP-AV node can only be determined after retrograde ERP of AP pathway is encountered.
ERP-HPS (panel D) is longest $V_{1} V_{2}$ where $V_{2}$ blocks below the His bundle recording site (i.e., HPS).

\section{RESULTS}

Anterograde and retrograde conduction and refractory period studies were performed in 22 consecutive patients with WPW Syndrome who had symptomatic PSVT. In only 14 of 22 patients was the PSVT inducible during right ventricular premature stimulation, and findings in these 14 cases form the basis of this report. In the remaining 8 of 22 cases, PSVT could not be elicited during ventricular premature stimulation for the reasons previously published (7). 12 of the 14 had ventricular preexcitation during sinus rhythm, whereas the remaining two (patients 5 and 8 ) had electrocardiographically concealed left sided AP. The pertinent clinical and electrophysiologic data are summarized in Table I. At the time of study all were in sinus rhythm and were not taking any cardioactive medications. Two patients had associated arteriosclerotic heart disease, and another two had mitral valve prolapse, whereas the remaining cases did not have clinically detectable structural heart disease. Although two or more basic ventricular cycle lengths were scanned in 10 of 14 patients, to simplify presentation of the results only the data from the shortest available cycle lengths in each case will be presented in detail.

Retrograde refractory period studies. Ventricular premature stimulation was initiated at $\mathrm{V}_{1} \mathrm{~V}_{2}$ intervals which measured 50-100 ms less than the basic drive, and the coupling intervals were progressively shortened. During the basic drive $\left(\mathrm{V}_{1}\right)$ six patients (patients $2,3,5,7,10$, and 13; Table I) demonstrated retrograde atrial depolarization exclusively via the AP, whereas fusion retrograde atrial activation was observed in the others (10). In the latter cases a change from fusion atrial activation to exclusive activation via the AP was noted as the $V_{1} V_{2}$ were further shortened. This change in retrograde atrial activation was noted at $V_{1} V_{2}$ intervals which had not yet produced emergence of a His deflection from the $\mathrm{V}_{2}$ electrogram. At the basic cycle length range of 550-900 ms (Table I), the His bundle deflection first emerged from $V_{2}$ at $V_{1} V_{2}$ intervals ranging from $320-500 \mathrm{~ms}$. Since in patients with retrogradely functioning AP the His bundle deflection following $\mathrm{V}_{2}$ could result from either retrograde conduction via the bundle branches or anterograde depolarization via the AV node following $A_{2}$ activation over the AP, the behavior of His bundle activation was closely analyzed (11). In all nine patients with left-sided (cases $1-9$, Table I) and 2 of 5 cases (patients 11 and 12) with right-sided VA AP, the separation of retrograde $\mathrm{A}_{2}$ from $\mathrm{V}_{2}$ deflection (on the His bundle electrogram tracing) was sufficient to provide a clear segment where the presence of a His deflection could be identified. In all 
A

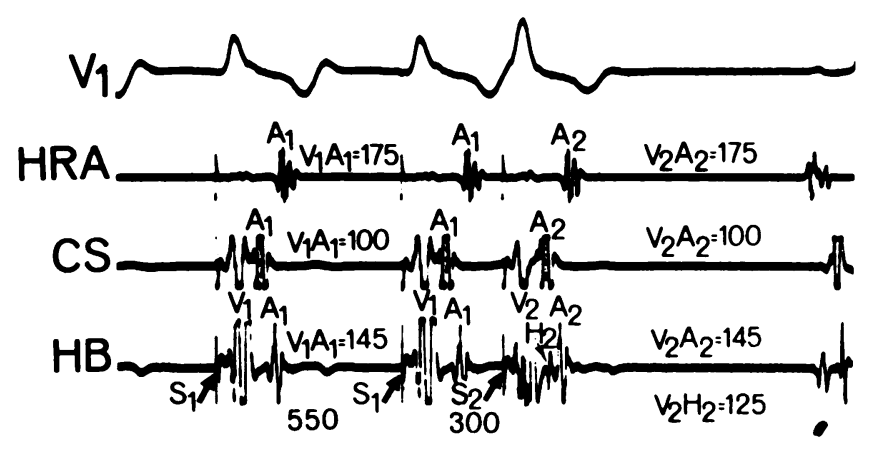

C

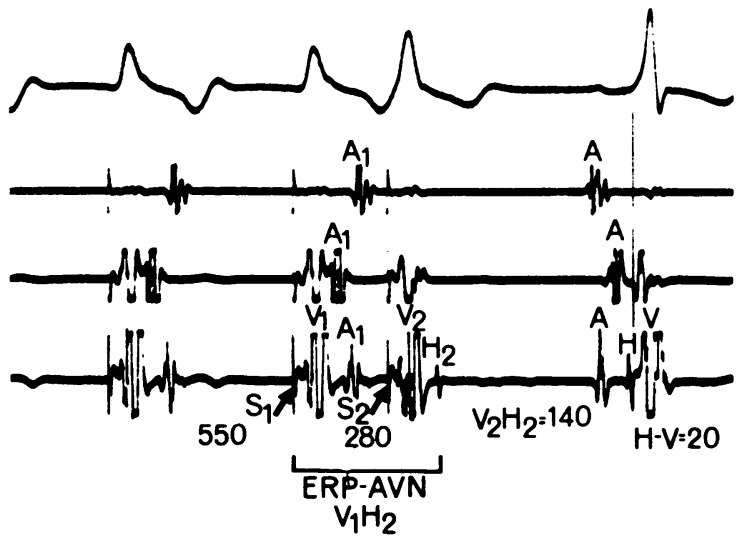

B
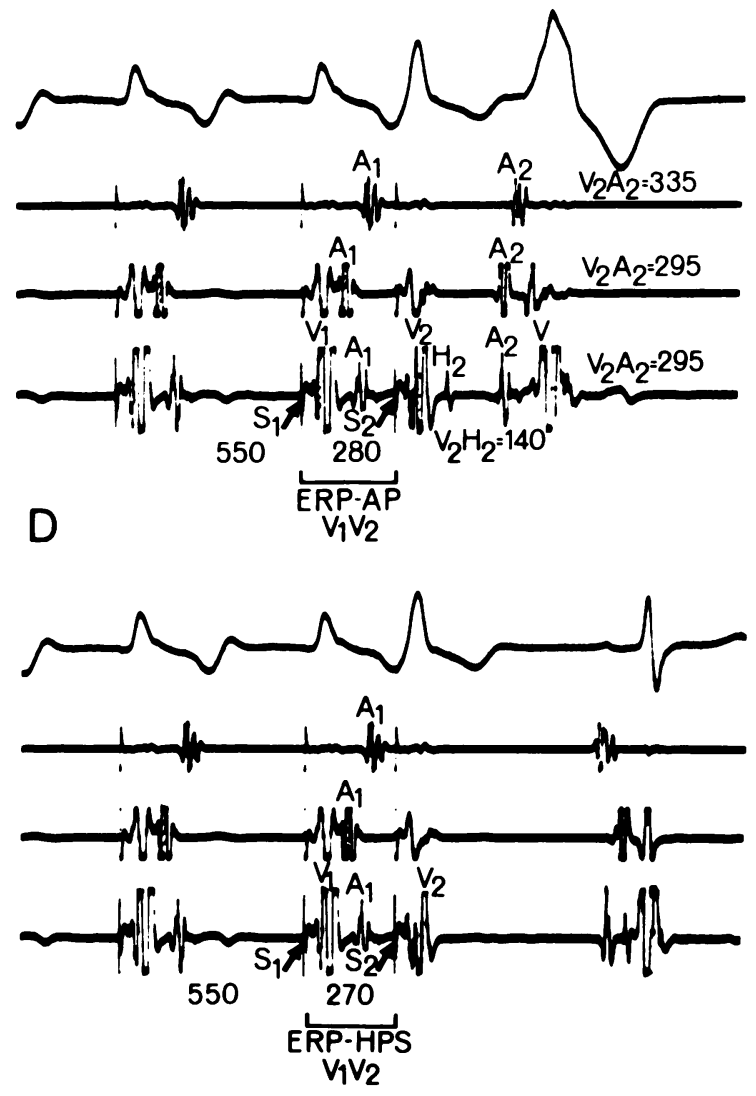

FIGURE 1 The basic right ventricular cycle length $\left(\mathrm{V}_{1} \mathrm{~V}_{1}\right)$ is constant at $550 \mathrm{~ms}$ in all panels, and progressively shorter $V_{1} V_{2}$ intervals are shown. Retrograde His bundle activation during the basic drive $\left(\mathrm{H}_{1}\right)$ is not identifiable, but $\mathrm{H}_{2}$ clearly follows $\mathrm{V}_{2}$ in panel $A$. The retrograde atrial activation during $\mathrm{V}_{1}$ as well as $\mathrm{V}_{2}$ occurs via $\mathrm{AP}$, as suggested by $(a)$ atrial activation sequence, and $(b)$ no change in $V_{2} A_{2}$ compared with $V_{1} A_{1}$ in any of the atrial electrograms, despite significant prolongation of HPS conduction time, i.e., $\mathrm{V}_{2} \mathrm{H}_{2}$ interval along the normal pathway. In panel $\mathrm{B}$, the $\mathrm{V}_{2}$ shows a retrograde block in the AP (ERP-AP) and exclusively activates the atria via HPS/AV node. Note the change in atrial activation sequence such that $\mathrm{A}_{2}$ electrograms on CS and $\mathrm{HBE}$ occur simultaneously. Subsequently, the $\mathrm{A}_{2}$ impulse conducts to the ventricles over the AP. In panel C, the $\mathrm{V}_{2}$ continues to block along the AP, but also shows a retrograde block in the $A V$ node. The longest $V_{1} H_{2}$ interval where a block is noted defines the AV nodal ERP. At a $V_{1} V_{2}$ of $270 \mathrm{~ms}$ (panel D) the $\mathrm{V}_{2}$ shows a retrograde block along both the AP and normal pathway. Unlike panel C, however, the site of block along the normal pathway is in the HPS (ERP-HPS). Perpendicular is drawn to indicate onset of QRS complex. Pertinent intervals are labeled. Anteriorly directed QRS complex as seen here is noted on occasions during right ventricular stimulation. $\mathrm{V}_{1}$, surface ECG Lead $V_{1}$; HRA, high right atrial electrogram; CS, coronary sinus electrogram; HBE, His bundle electrogram.

of these 11 patients the His bundle deflection preceded the retrograde $\mathrm{A}_{2}$ deflection (Figs. 2 and 3) during its initial appearance, indicating retrograde His bundle activation via the bundle branches $\left(\mathrm{H}_{2}\right)$; the designation of $\mathrm{H}_{2}$ henceforth will imply such an occurrence. With further shortening of the $\mathrm{V}_{1} \mathrm{~V}_{2}$ intervals, the $\mathrm{V}_{2} \mathrm{H}_{2}$ delays showed almost linear increases as long as the retrograde $\mathrm{H}_{2}$ deflection was still identifiable and preceded or coincided with the retrograde $A_{2}$ electrogram (Figs. 2 and 3). This relationship between $V_{1} V_{2}$ and $\mathrm{V}_{2} \mathrm{H}_{2}$ intervals is typical of retrograde $\mathrm{H}_{2}$ activation via the bundle branches and has been previously demonstrated in patients without the WPW Syndrome $(9,12-15)$.

At the $V_{1} V_{2}$ intervals that produced emergence of retrograde $\mathrm{H}_{2}$ deflection, the $\mathrm{V}_{2} \mathrm{~A}_{2}$ intervals via the AP showed no further prolongation once exclusive $V_{2} A_{2}$ conduction via the AP was previously established (Figs. 2-4) at longer coupling intervals. Upon further shortening of the ventricular coupling intervals in 8 of 


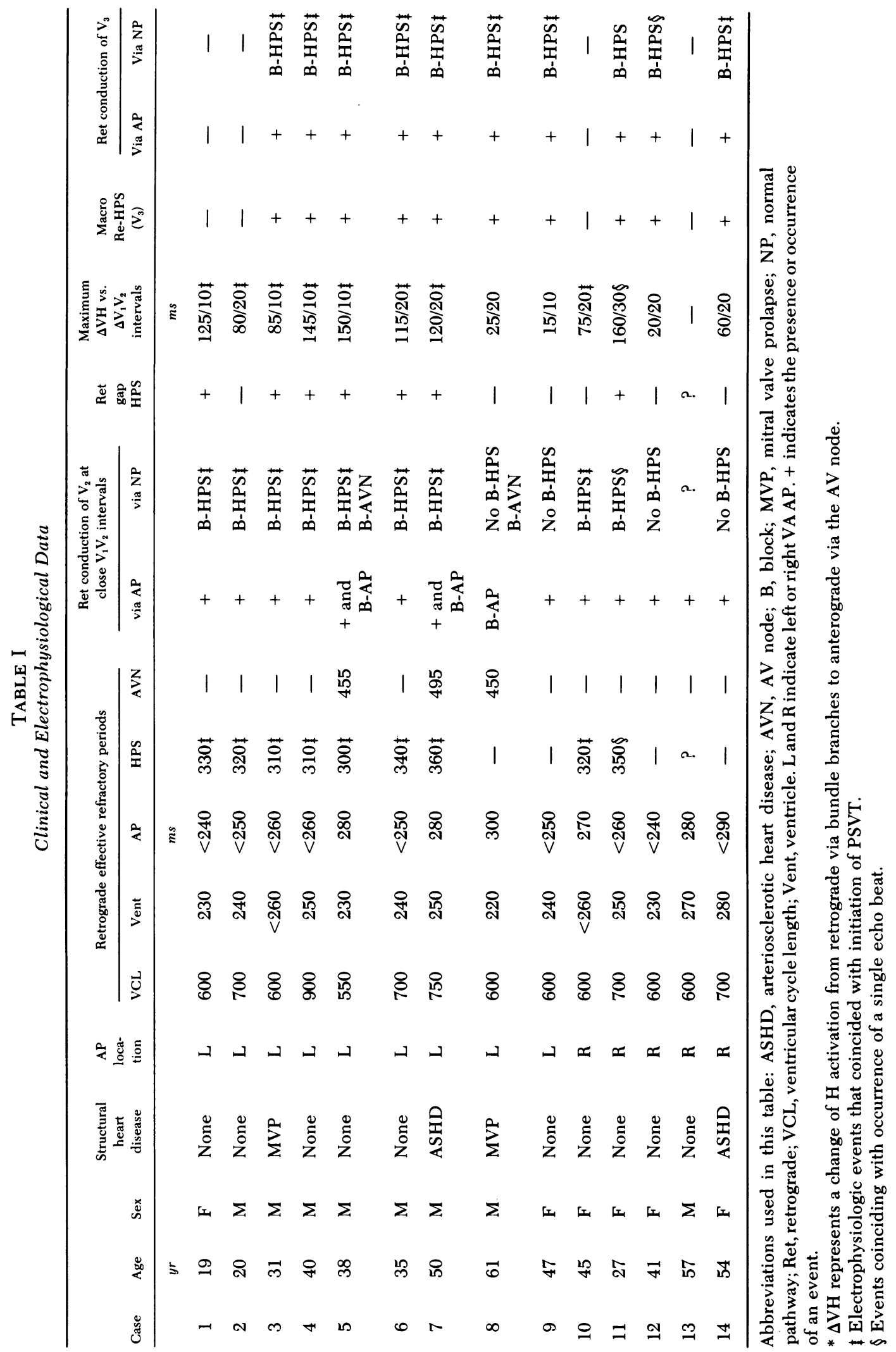


A

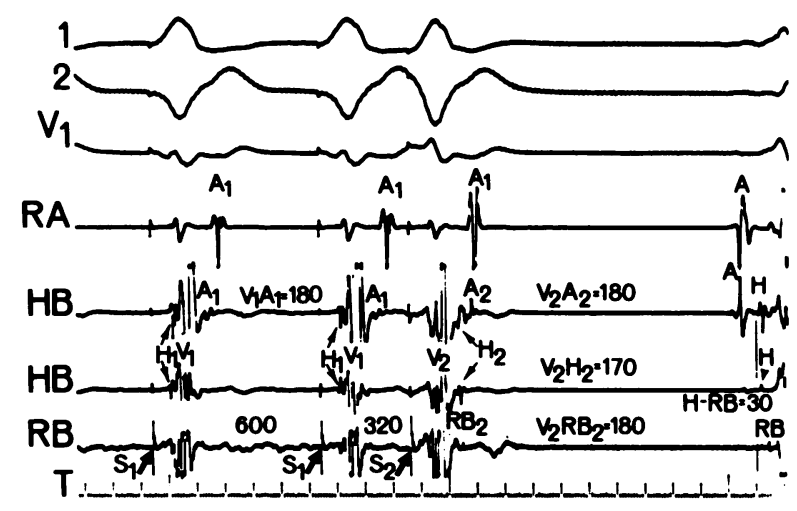

C

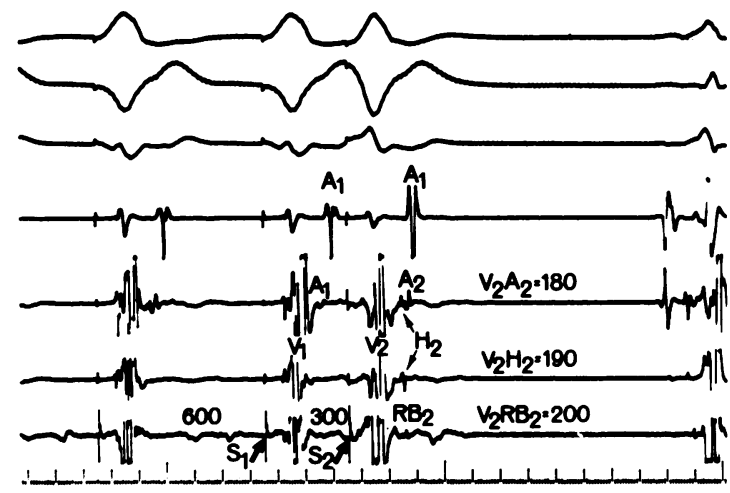

B

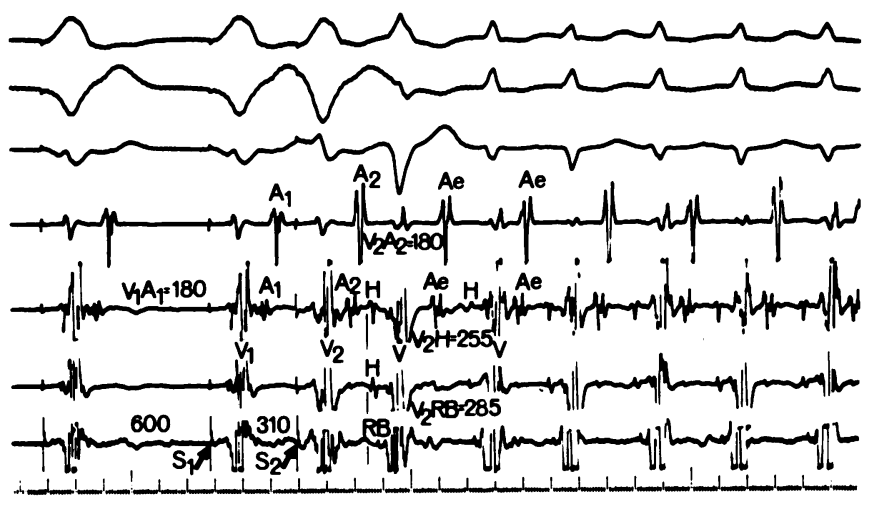

D

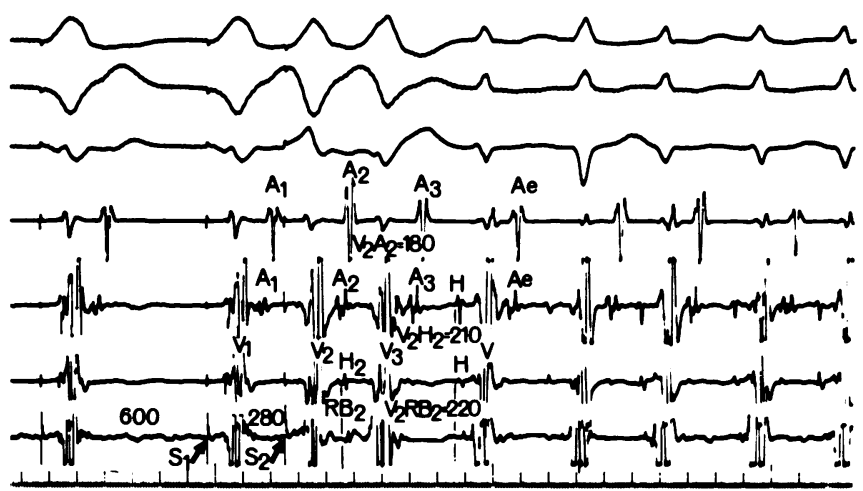

Figure 2 (Patient 3) Basic ventricular cycle length is constant at $600 \mathrm{~ms}$ (panels A-D). The $V_{1} A_{1}$ and $V_{2} A_{2}$ intervals measure the same in all panels despite progressive shortening in the $V_{1} V_{2}$ intervals. In addition to the two $H B$ tracings, a recording from the $R B$ was also obtained with the H-RB interval measuring a $30 \mathrm{~ms}$ during sinus rhythm. The $\mathrm{V}_{2}$ in panel A depolarizes the $\mathrm{H}_{2}$ via the left bundle branch (LBB) as suggested by $\mathrm{H}_{2}-\mathrm{RB}_{2}$ interval of $10 \mathrm{~ms}$. A 10-ms shortening in $\mathrm{V}_{1} \mathrm{~V}_{2}$ interval (panel $\mathrm{B}$ ) results in an increase in the $\mathrm{V}-\mathrm{H}$ from $170 \mathrm{~ms}$ in panel A to $225 \mathrm{~ms}$ in panel $B$, and PSVT is initiated. Note that the His deflection in panel $B$ follows $A_{2}$, and $H-R B$ interval equals that of sinus beats $(30 \mathrm{~ms})$ indicating $H i s$ bundle activation via the AV node. The $\mathrm{A}_{2} \mathrm{H}$ interval equals sinus AH (not labeled) and is significantly shorter compared with Ae-H of PSVT, suggesting lack of retrograde penetration of the AV node by $V_{2}$ impulse. The QRS following His deflection shows an LBB block (B) pattern, indicating incomplete recovery of LBB following $V_{2}$ despite retrograde bilateral block HPS. At shorter $V_{1} V_{2}$ of $300 \mathrm{~ms}$ (panel $C$ ) $V_{2} H_{2}$ conduction via the LBB resumes (retrograde gap HPS) and PSVT is not induced. Retrograde $\mathrm{H}_{2}$ activation via the LBB continues in panel $D$ at a shorter coupling interval, and a $V_{3}$ due to macroreentry HPS is noted. The $V_{3}$ produces $A_{3}$ response via the AP blocks in the HPS, and a PSVT is started. 1,2 , and $V_{1}$, surface ECG leads; RA, right atrial electrogram; RB, right bundle electrogram tracing; $\mathrm{T}$, time lines.

these 11 cases (cases 1-7 and 11, Table I), the His deflection suddenly shifted from a location preceding or coinciding with the $A_{2}$ (on $\mathrm{HB}$ electrocardiogram) to a position following the $A_{2}$ (Figs. 2-4). This change in location of His bundle deflection was accompanied by an increase in $\mathrm{V}$-H interval ranging from 40 to $155 \mathrm{msec}$ (mean, 121.5 \pm 29.1 ) for a $10-30 \mathrm{~ms}$ (mean, 16.2 \pm 7.4 ) decrease in the $\mathrm{V}_{1} \mathrm{~V}_{2}$ interval (Table $\mathrm{I}$ ). The succeeding $\mathrm{A}_{2} \mathrm{H}$ intervals in most cases measured equal to or were slightly longer than (i.e., $10-20 \mathrm{~ms}$ ) the $\mathrm{A}-\mathrm{H}$ intervals of sinus beats (except in patient 11) but significantly shorter as compared with the A-H intervals during PSVT. In all of these eight cases ventricular activation followed the His bundle activation, which in turn was followed by retrograde atrial activation via the AP (atrial echo beat or Ae), and PSVT was initiated (Figs. $2-4$ ) in all except one case (case 11). In case 11 the Ae beat anterogradely blocked in the AV node and no sustained PSVT occurred. The sudden increase in the V-H interval was not due to retrograde block in the right bundle branch and subsequent retrograde $\mathrm{His}$ activation via the left bundle branch. The events can be best 
A
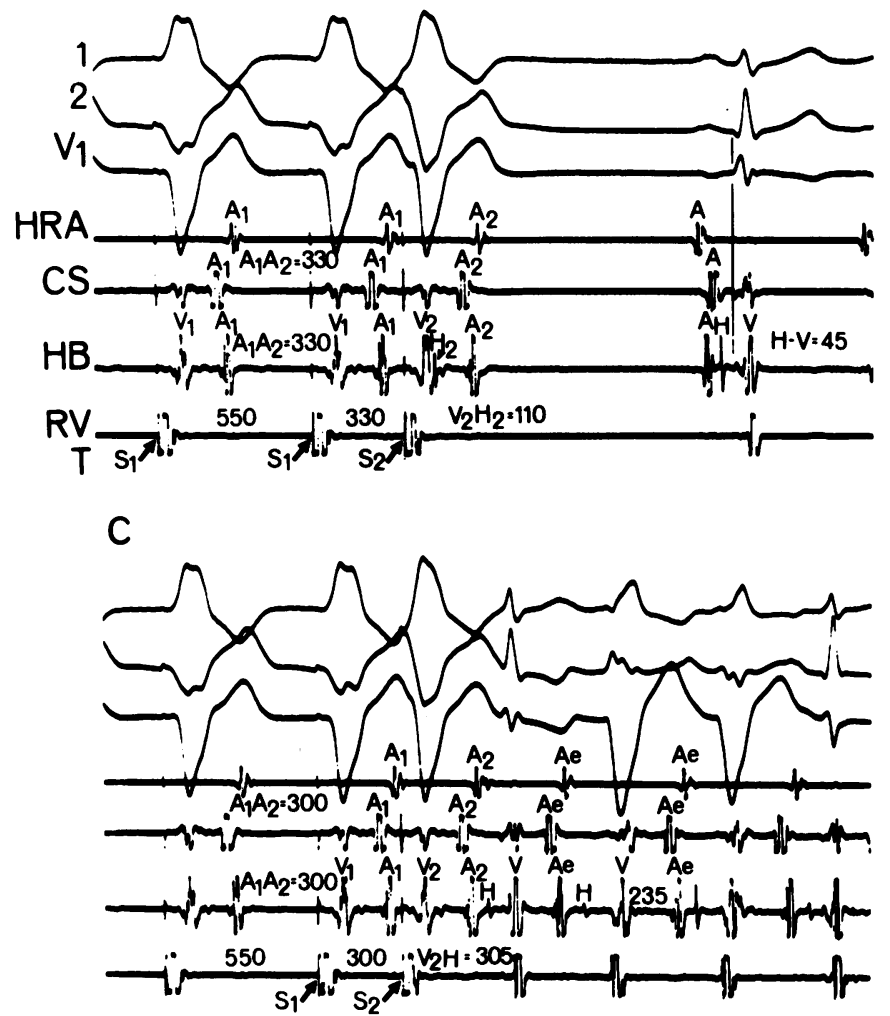

B

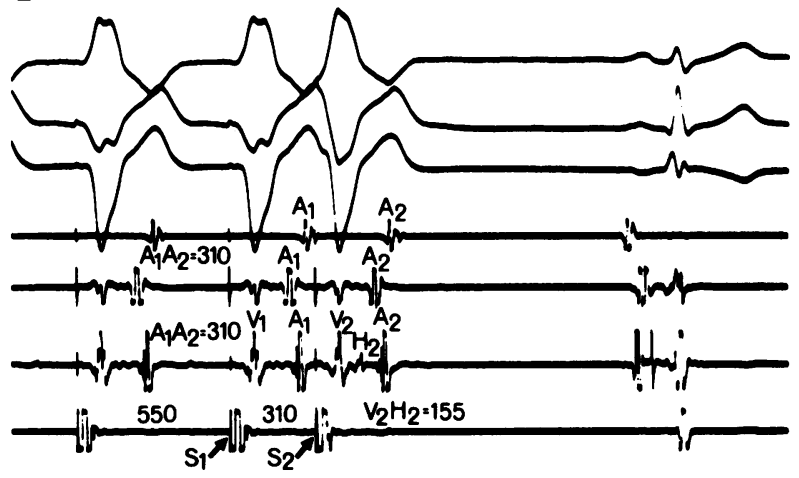

D

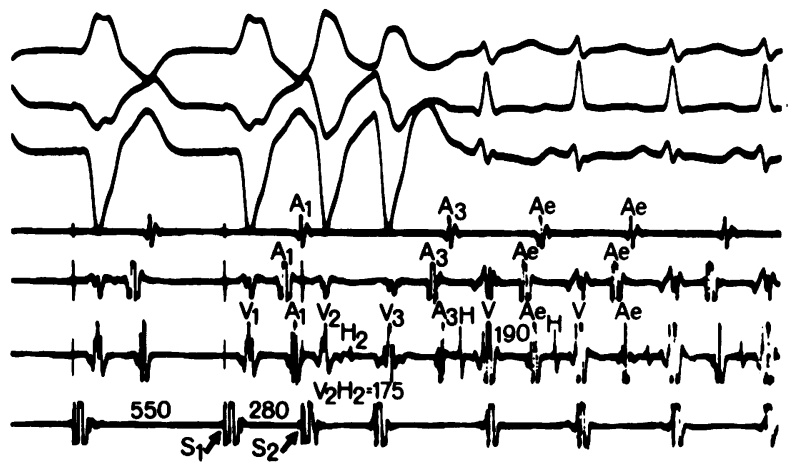

Figure 3 (Patient 5, concealed left-sided AP) The basic ventricular cycle length is constant and measures $550 \mathrm{~ms}$ in all panels. Exclusive VA conduction via the AP is noted during the basic drive and premature beats in panels $\mathrm{A}-\mathrm{C}$. Note the absence of ventricular preexcitation and normal $\mathrm{H}-\mathrm{V}$ intervals during sinus beats. An increase in $\mathrm{V}_{2} \mathrm{H}_{2}$ interval is noted from panels $A-B$, the $\mathrm{H}_{2}$ preceding $A_{2}$ on $H B$ electrogram. In panel $C$, while the $V_{2} A_{2}$ is unchanged, the His potential now follows the $A_{2}$, and PSVT is initiated. A 10 -ms decrease in $V_{1} V_{2}$ (from 310 in panel $B$ to 300 in panel C) produces a 150 -ms increase in the $\mathrm{VH}$ interval from 155 to $305 \mathrm{~ms}$ ) and the $\mathrm{A}_{2} \mathrm{H}$ interval is only slightly longer than $\mathrm{AH}$ of the sinus beats (AH intervals not labeled), both of which suggest that the $\mathrm{V}_{2}$ in panel $\mathrm{C}$ retrogradedly blocked in the HPS and the His deflection represents anterograde activation from the $A_{2}$ impulse. This is further supported by resumption of $\mathrm{V}_{2} \mathrm{H}_{2}$ conduction $\left(\mathrm{V}_{2} \mathrm{H}_{2}-175 \mathrm{~ms}\right.$, panel D) at shorter $\mathrm{V}_{1} \mathrm{~V}_{2}$ intervals of $280 \mathrm{~ms}$. In panel D no PSVT is initiated by $\mathrm{V}_{2}$ and the impulse retrogradely blocks in the AP as well as the AV node. However, the $\mathrm{H}_{2}$ is followed by macroreentry HPS $\left(V_{3}\right)$, which in turn shows a retrograde block HPS while activating atria $\left(A_{3}\right)$ via the AP. Anterograde propagation of $A_{3}$ starts the PSVT. Note that the V-Ae measures $235 \mathrm{~ms}$ (panel C) during LBB block and 190 (panel D) during normal intraventricular conduction, findings suggestive of left-sided VA AP. RV, right ventricular electrogram.

explained as follows: The $\mathrm{V}_{2}$ retrogradely blocked bilaterally in the HPS (i.e., no $\mathrm{H}_{2}$ ), yet reached the atrium via the AP. Since the AV node was not penetrated by the $V_{2}$ impulse, the $A_{2}$ response was followed by relatively fast anterograde $\mathrm{AV}$ nodal conduction, i.e., $\mathrm{A}_{2} \mathrm{H}$ interval. The longer $\mathrm{H}-\mathrm{V}$ intervals and the aberrant conduction of the ensuing QRS complex noted in some cases was due to retrograde concealed conduction of $\mathrm{V}_{2}$ in the HPS. The reasons for the above assumption, which was directly documented in two cases by simultaneous His and right bundle (RB) recordings (Fig. 2), will be discussed later. To simplify presentation of the results, the designation $\mathrm{V}_{2} \mathrm{H}$ will imply anterograde depolarization of the His potential via the AV node following retrograde $A_{2}$ activation over the AP. The occurrence of these electrophysiologic events in individual cases, i.e., retrograde block in the HPS and a shift in His activation from retrograde via the bundle branches to anterograde via the AV node and initiation of PSVT, is depicted in the table. In the remaining 3 of 11 cases $(8,9$, and 12$)$, a retrograde block in the HPS was not noted and $\mathrm{V}_{2}$ continued to activate $\mathrm{H}_{2}$ via the bundle branches, as indicated by the following: $(a)$ persistent linear relationship between $\mathrm{V}_{2} \mathrm{H}_{2}$ and $\mathrm{V}_{1} \mathrm{~V}_{2},(b)$ the His deflection never showed a shift to a position succeeding the $A_{2}$ electrogram. None of these three cases demon- 


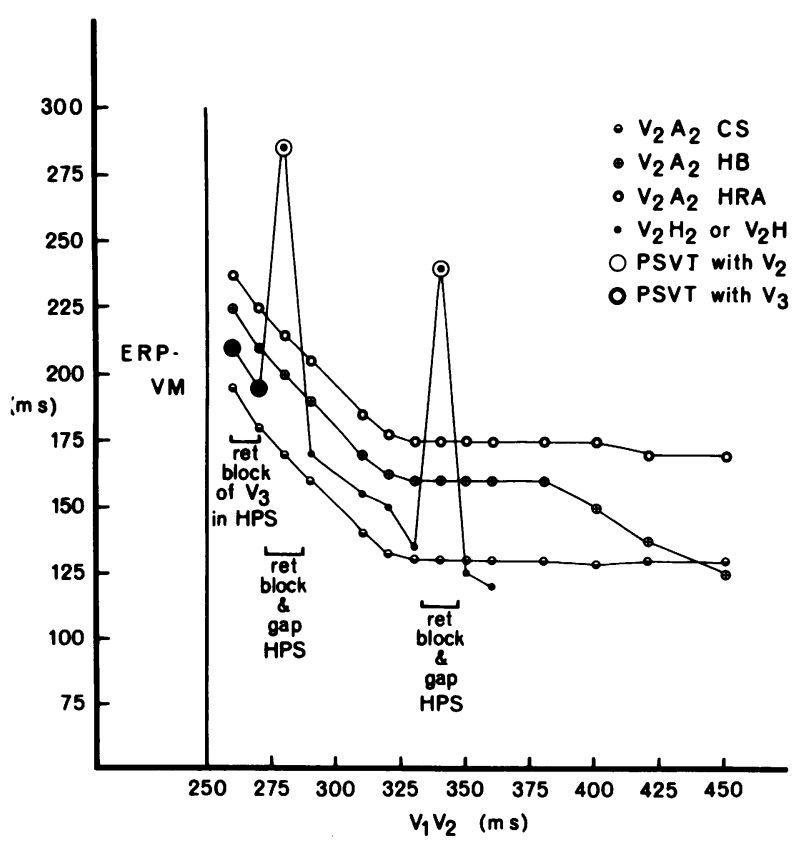

Figure 4 (Patient 6) A typical sequence of events during right ventricular premature stimulation is depicted. At a basic ventricular cycle length of $700 \mathrm{~ms}$ retrograde $A_{2}$ activation initially occurs via both the normal pathway and AP at $V_{1} V_{2}$ of $450 \mathrm{~ms}$, suggesting fusion activation of the atria. Note that with progressive shortening in the $V_{1} V_{2}$, the $V_{2} A_{2}$ on $C S$ electrogram shows no change; $\mathrm{V}_{2} \mathrm{~A}_{2}$ on HRA tracings shows slight increase, whereas $\mathrm{V}_{2} \mathrm{~A}_{2}$ on $\mathrm{HB}$ electrogram displays a distinct increase. Exclusive $V_{2} A_{2}$ conduction via the $A P$ is noted at $V_{1} V_{2}$ of $380 \mathrm{~ms}$, and at closer $V_{1} V_{2}$ intervals the $V_{2} A_{2}$ intervals show a parallel increase on all atrial electrogram tracings. Retrograde $\mathrm{H}_{2}$ activation is first noted at a $V_{1} V_{2}$ of $360 \mathrm{~ms}$, and it precedes $\mathrm{A}_{2}$ deflections on all atrial electrograms. A sudden increase in VH interval occurs at a coupling interval of $340 \mathrm{~ms}$ due to retrograde block of $\mathrm{V}_{2}$ in the HPS, and a PSVT is initiated. $\mathrm{V}_{2} \mathrm{H}_{2}$ conduction resumes between $V_{1} V_{2}$ of 330-290 ms (retrograde gap HPS), and PSVT can no longer be started. Upon further shortening in $V_{1} V_{2}$ to 280 $m s$ the $V_{2}$ again demonstrates a retrograde block in the HPS with resulting PSVT. Resumption of $\mathrm{V}_{2} \mathrm{H}_{2}$ conduction at $\mathrm{V}_{1} \mathrm{~V}_{2}$ of 270-260 ms is associated with occurrence of $V_{3}$ due to macroreentry HPS, which in turn starts the PSVT. The $V_{3} A_{3}$ conduction occurs via AP and is not depicted. Note that with $V_{1} V_{2}$ up to coupling interval of $330 \mathrm{~ms}$, the $V_{2} H_{2}$ delays were accompanied by constant $V_{2} A_{2}$ conduction via the AP; the latter showed delays only at $V_{1} V_{2}<320 \mathrm{~ms}$. Also, when $V_{2}$ activated the $\mathrm{H}_{2}$ via the bundle branches-i.e., when $\mathrm{H}_{2}$ preceded $\mathrm{A}_{2}$ on HBE- no PSVT could be initiated. ERP-VM, effective refractory period-ventricular myocardium.

strated initiation of PSVT or an Ae response following $\mathrm{V}_{2}$.

In two patients (10 and 14) with right-sided VA AP, the onset of low atrial $A_{2}$ was inscribed within the local $\mathrm{V}_{2}$ electrogram, and the $\mathrm{His}$ deflection followed the $A_{2}$ the very first time it emerged from $V_{2}$. Therefore, it was difficult to determine if the His deflection represented anterograde or retrograde activation. In case 10 , how- ever, a sudden increase in the $\mathrm{V}-\mathrm{H}$ of $75 \mathrm{~ms}$ with a $20 \mathrm{~ms}$ decrease in $V_{1} V_{2}$ coincided with initiation of PSVT, suggesting that the initial His activation was retrograde, whereas the onset of PSVT heralded a retrograde block in the HPS, findings similar to all other cases where $\mathrm{V}_{2}$ initiated PSVT.

In the one remaining patient (13), at no time did the His deflection precede $A_{2}$, and a change from retrograde to anterograde depolarization of the His bundle could not be documented. Although a PSVT was initiated in this case by $V_{2}$, discernment of the exact mechanism was not certain.

Retrograde gap phenomenon HPS (16). Following the initial retrograde block of $V_{2}$ in the HPS, further shortening in $V_{1} V_{2}$ intervals resulted in resumption of retrograde conduction to the $\mathrm{His}$ bundle (i.e., $\mathrm{V}_{2} \mathrm{H}_{2}$ ) in seven of nine cases $(1,3-7$, and 11 , Table I retrograde gap phenomenon HPS), as was suggested by return of $\mathrm{H}_{2}$ deflection preceding or coinciding with the $\mathrm{A}_{2}$ electrogram (Figs. 3 and 4). The $\mathrm{V}_{2} \mathrm{H}_{2}$ values following resumption of conduction were in line with those expected from a reciprocal relationship between $V_{1} V_{2}$ and $\mathrm{V}_{2} \mathrm{H}_{2}$ intervals and significantly shorter than the $\mathrm{V}_{2} \mathrm{H}$ values noted at longer coupling intervals (Fig. 4). Return of $\mathrm{V}_{2} \mathrm{H}_{2}$ conduction occurred while retrograde $A_{2}$ activation persisted via the AP in all cases except one (point No. 5, Fig. 3), where a concomitant retrograde block was noted along the AP. Whenever retrograde $\mathrm{H}_{2}$ followed $V_{2}$ with or without associated $A_{2}$ activation via the AP, the Ae or PSVT was not initiated by $V_{2}$ either before or after the gap zone in any of the cases in this series.

Macroreentry HPS $(12,13) .10$ of the 14 cases (3-9, 11,13 , and 14, Table I) demonstrated occurrence of macroreentry HPS in the form of $\mathrm{V}_{3}$ following retrograde $\mathrm{H}_{2}$ activation. The $\mathrm{V}_{3}$ in turn showed a retrograde block in the HPS along the normal pathway (i.e., no $\mathrm{H}_{3}$ ) in all patients (Fig. 3). The $V_{3}$, however, conducted to the atrium $\left(\mathrm{A}_{3}\right)$ via the $\mathrm{AP}$ in all cases and an $\mathrm{Ae}$ or PSVT was started in 9 of 10 (Table I, Fig. 3). In the remaining one case the $A_{3}$ blocked in the AV node, and no Ae or PSVT was noted.

Findings at other basic cycle lengths. In 10 of 14 cases, two or more basic cycle lengths were tested, and the results were similar to those presented above, i.e., the PSVT was only initiated when $V_{2}$ and/or $V_{3}$ retrogradely blocked in the HPS while still activating atrial via the AP.

\section{DISCUSSION}

Results of this study indicate that retrograde HPS block of $V_{2}$ and/or $V_{3}$ in the HPS is a relatively common mechanism by which PSVT is initiated during right ventricular premature stimulation in patients with the WPW Syndrome (13 of 14 cases in this series). Although 
the induced $V_{2}$ may initiate PSVT by other mechanisms, present data suggest that other mechanisms are less commonly responsible for PSVT initiation during ventricular premature stimulation. In a series with 139 cases of WPW Syndrome reported by Wellens (6) it was noted that, during ventricular premature stimulation, a PSVT was most commonly initiated when $V_{2}$ retrogradely blocked along the normal pathway (30 of 139 cases) or by $V_{3}$ ( 9 of 139 cases) when the latter was inducible, and by other mechanisms in only 11 of 139 cases. The exact site of retrograde block (HPS vs. AV node) of $V_{2}$ or $V_{3}$ was not demonstrated, and it cannot be stated with certainty whether the findings in the report by Wellens and the present study are comparable. A recent study showed that in patients with ventricular preexcitation, programmed ventricular extrastimuli did not initiate PSVT if there was no VA conduction and there was persistent retrograde atrial fusion activation via both the AP and normal pathway (7). A narrow QRS PSVT could be elicited with $V_{2}$ when VA conduction was intact, $\mathrm{V}_{2} \mathrm{~A}_{2}$ conduction occurred exclusively via the AP, and normal pathway was less deeply penetrated (7). In that report it was indicated, however, that the level or site of retrograde block along the normal pathway could not be determined.

Regular narrow QRS complex PSVT is the most common arrhythmia elicited by programmed atrial as well as ventricular premature stimulation in patients with the WPW Syndrome $(3,4)$. During premature atrial stimulation $\left(A_{2}\right)$, the premature impulse blocks in the AP, activates the ventricles over the AV node-HPS, and returns to the atria via the AP to start the PSVT. Induction of the same PSVT with $\mathrm{V}_{2}$, on the other hand, will be more likely if $\mathrm{V}_{2} \mathrm{~A}_{2}$ conduction occurred via the $\mathrm{AP}$ and subsequent anterograde propagation took place along the AV node-HPS. The latter occurrence will obviously be facilitated by a retrograde block of $V_{2}$ in the normal pathway. A retrograde block of $V_{2}$ above the His bundle (AV node), however, may not permit initiation of PSVT even if accompanied by concomitant retrograde $\mathrm{A}_{2}$ activation via the AP, because of effect of retrograde concealed conduction in the AV node. Under the following circumstances, however, $\mathrm{V}_{2}$ may start PSVT despite AV nodal penetration $(a)$ when $\mathrm{V}_{2}$ produces only a partial penetration of the $\mathrm{AV}$ node with resultant rapid recovery of excitability, $(b)$ initiates intranodal reciprocation, $(c)$ conducts with long $\mathrm{V}_{2} \mathrm{~A}_{2}$ via $\mathrm{AP}$ sufficient to allow recovery of $\mathrm{AV}$ nodal excitability. A retrograde block of $V_{2}$ in the HPS, on the other hand, would permit unhindered progress of $A_{2}$ impulse through the $A V$ node, since the prior $A V$ nodal depolarization is during the last $V_{1}$.

A support for the preceding hypothesis is provided in the present study by demonstration that the PSVT initiation did coincide with the occurrence of retro- grade block of $\mathrm{V}_{2}$ in the HPS. That the site of retrograde block of $V_{2}$ was indeed in the HPS rather than the AV node is suggested by the following observations: (A) A sudden increase in the $\mathrm{V}-\mathrm{H}$ values (a mean increase of $121.8 \mathrm{~ms}$ from $\mathrm{V}_{2} \mathrm{H}_{2}$ to $\mathrm{V}_{2} \mathrm{H}$, for a $16.2 \mathrm{~ms}$ mean decrease) in $\mathrm{V}_{1} \mathrm{~V}_{2}$ intervals, a behavior unlike that of retrograde $\mathrm{H}_{2}$ activation via the bundle branches and therefore suggesting a shift from retrograde to anterograde activation of the His bundle (Figs. 2-4). (B) A return of $\mathrm{H}_{2}$ deflection at shorter $\mathrm{V}_{1} \mathrm{~V}_{2}$, producing $\mathrm{V}_{2} \mathrm{H}_{2}$ values less than $\mathrm{V}_{2} \mathrm{H}$ values (retrograde gap phenomenon HPS) noted at longer $\mathrm{V}_{1} \mathrm{~V}_{2}$, and concomitant abolition of PSVT (Figs. 3 and 4). (C) Direct demonstration of the shift from a retrograde to anterograde activation of the His bundle when both $\mathrm{His}$ and RB recordings were available (two cases, Fig. 2). (D) The occurrence of macroreentry HPS after retrograde $\mathrm{H}_{2}$ activation and its disappearance despite longer V-H intervals, coinciding with the shift of $\mathrm{His}$ deflection to a location succeeding the $A_{2}$ (Fig. 3). (E) $A$ consistent relationship between the $A_{2}$ and the succeeding His deflection with values approximating the sinus A-H values. This suggests that the occurrence of His deflection after $\mathrm{A}_{2}$ was not an independent phenomenon (retrograde via bundle branches) but its occurrence was directly related to and in fact dependent upon the preceding $A_{2}$ activation (Figs. 2 and 3).

It seems reasonable to conclude, therefore, that the His deflection following the $A_{2}$ was due to anterograde depolarization via the AV node. Despite the above reasoning, questions can be raised as to whether the $V_{2}$ might still have blocked in the AV node rather than HPS, and whether the $\mathrm{H}_{2}$ deflection was either not identifiable for some reason or was obscured by the local $\mathrm{V}_{2}$ electrogram. These questions can be dismissed for the following reasons: $(a)$ Once the retrograde $\mathrm{H}_{2}$ deflection emerges, the $\mathrm{V}_{2} \mathrm{H}_{2}$ intervals generally lengthen (rather than shorten) at shorter $\mathrm{V}_{1} \mathrm{~V}_{2}(9,12,14$, 15). (b) The occurrence of gap phenomenon in most cases suggests retrograde $\mathrm{H}_{2}$ was recordable whenever the His bundle was depolarized. (c) At the $\mathrm{V}_{2} \mathrm{H}$ interval ranges noted in these cases, the $\mathrm{His}$ bundle activation will have to occur twice (first retrogradely and then anterogradely) within a period of $300 \mathrm{~ms}$, an unlikely occurrence. In the event the $V_{2}$ retrogradely penetrates the $\mathrm{AV}$ node, the subsequent $\mathrm{A}_{2} \mathrm{H}$ intervals will be expected to be much longer than the sinus beats (retrograde concealed conduction in the AV node). Such a mechanism of PSVT initiation was not noted in the present series but has been previously described, and may operate more frequently at shorter cycle lengths due to abbreviation in the refractoriness of the His. For similar reasons a retrograde AV nodal block of ventricular impulse may also 
be a more common mechanism of PSVT initiation during rapid incremental ventricular pacing rather than premature stimulation (8).

It was repeatedly observed during this study that whenever $\mathrm{V}_{2}$ produced a retrograde $\mathrm{H}_{2}$ response, the PSVT could not be initiated. Since concomitant retrograde $A_{2}$ activation in most instances took place exclusively via the AP, the collision of the two impulses via the normal pathway and AP must have occurred in the AV node rather than the atrium $(7,17)$. Retrograde penetration and concealed conduction in the AV node was most probably responsible for preventing the forward progress of the $A_{2}$ impulse. It is easily understandable, therefore, why the onset of retrograde block in the HPS coincided with initiation of a PSVT or an Ae response. It may also be pointed out that the response of HPS and AP to $V_{2}$ was not significantly different between right- and left-sided AP, aside from the fact that the location of $A_{2}$ electrogram in patients with right VA AP made analysis of events more difficult.

When an $\mathrm{H}_{2}$ response followed $\mathrm{V}_{2}$, the PSVT only occurred if $\mathrm{V}_{3}$ followed. Although in this group of patients the $V_{3}$ phenomenon occurred dur to macroentry HPS, $a V_{3}$ from other mechanisms - local reentry, etc. - will be expected to produce the same results (13). The initiation of an Ae response or PSVT following $V_{3}$ was also facilitated by a retrograde block of $V_{3}$ in the HPS and concomitant retrograde $A_{3}$ activation via the AP, as was noted in 9 of 14 cases in this series.

\section{ACKNOWLEDGMENT}

The authors thank Kathryn Corriere and Robert Walters for their assistance.

\section{REFERENCES}

1. Durrer, D., L. Schoo, R. M. Schuilenburg, and H. J. J. Wellens. 1967. Role of premature beats in the initiation and the termination of supraventricular tachycardia in the Wolff-Parkinson-White Syndrome. Circulation. 36: 644-662.

2. Wellens, H. J. J., D. R. Düren, and K. I. Lie. 1976. Observations on mechanisms of ventricular tachycardia in man. Circulation. 54: 237-244.

3. Gallagher, J. J., M. Gilbert, R. H. Svenson, W. C. Sealy, J. Kasell, and A. G. Wallace. 1975. Wolff-Parkinson-White Syndrome. The problem, evaluation, and surgical consideration. Circulation. 51: 767-784.

4. Wellens, H. J. J., and D. Durrer. 1975. The role of an accessory atrio-ventricular pathway in reciprocating tachycardia. Observations in patients with and without the Wolff-Parkinson-White Syndrome. Circulation. 52: 58-72.

5. Wellens, H. J., and D. Durrer. 1974. Patterns of ventriculoatrial conduction in the Wolff-Parkinson-White Syndrome. Circulation. 49: 22-31.

6. Wellens, H. J. J. 1977. Modes of initiation of circus movement tachycardia in 139 patients with the WolffParkinson-White Syndrome studied by programmed electrical stimulation. In Re-entrant Arrhythmias. Mechanisms and Treatment. H. E. Kulbertus, editor. MTP Press Limited, Lancaster. 153-169.

7. Sung, R. J., A. Castellanos, S. M. Mallon, H. Gelband, I. Mendoza, and R. J. Myerburg. 1977. Mode of initiation of reciprocating tachycardia during programmed ventricular stimulation in the Wolff-Parkinson-White Syndrome with reference to various patterns of ventricular conduction. Am. J. Cardiol. 40: 24-31.

8. Denes, P., D. Wu, F. Amat-Y-Leon, R. Dhingra, R. Bauernfeind, R. Kehoe, and K. M. Rosen. 1978. Determinants of atrioventricular reentrant paroxysmal tachycardia in patients with Wolff-Parkinson-White Syndrome. Circulation. 58: 415-425.

9. Akhtar, M., A. N. Damato, W. P. Batsford, J. N. Ruskin, and J. B. Ogunkelu. 1975. A comparative analysis of anterograde and retrograde conduction patterns in man. Circulation. 52: 766-778.

10. Svenson, R. H., H. C. Miller, J. J. Gallagher, and A. G. Wallace. 1975. Electrophysiological evaluation of the Wolff-Parkinson-White Syndrome: problems in assessing anterograde and retrograde conduction over the accessory pathway. Circulation. 52: 552-562.

11. Akhtar, M., A. N. Damato, J. N. Ruskin, W. P. Batsford, C. P. Reddy, A. R. Ticzon, M. S. Dhatt, J. A. C. Gomes, and A. H. Calon. 1978. Antegrade and retrograde conduction characteristics in three patterns of paroxysmal atrioventricular junctional reentrant tachycardia. Am. Heart J. 95: 22-42.

12. Akhtar, M., A. N. Damato, W. P. Batsford, J. B. Ogunkelu, and J. N. Ruskin. 1974. Demonstration of re-entry within the His-Purkinje system in man. Circulation. 50: 11501162.

13. Akhtar, M., C. Gilbert, F. Wolf, and D. Schmidt. 1978. Reentry within the His Purkinje system. Elucidation of reentrant circuit utilizing right bundle branch and His bundle recordings. Circulation. 58: 295-304.

14. Akhtar, M., C. J. Gilbert, F. G. Wolf, and D. H. Schmidt. 1979. Retrograde conduction in the His-Purkinje system: analysis of the routes of impulse propagation using $\mathrm{His}$ and right bundle branch recordings. Circulation. 59: 1252-1265.

15. Josephson, M. E., and J. A. Kastor. 1978. His-Purkinje conduction during retrograde stress. J. Clin. Invest. 61: $171-177$.

16. Akhtar, M., A. N. Damato, A. R. Caracta, W. P. Batsford, and S. H. Lau. 1974. The gap phenomena during retrograde conduction in man. Circulation 49: 811-817.

17. Akhtar, M., C. J. Gilbert, M. Al-Nouri, and D. H. Schmidt. 1979. Electrophysiologic mechanisms for modification and abolition of atrioventricular junctional tachycardia with simultaneous and sequential atrial and ventricular pacing. Circulation. 60: 1443-1454. 